\title{
Single centre 12 year experience with durable mechanical circulatory support: comparison with the EUROMACS registry
}

\author{
Hulman $\mathrm{M}^{1}$, Ondrusek $\mathrm{M}^{1}$, de By $\mathrm{TMMH}^{2}$, Antonides $\mathrm{CFJ}^{3}$, Artemiou $\mathrm{P}^{1}$, Hudec $\mathrm{V}^{1}$, \\ Gasparovic $\mathrm{I}^{1}$, Lesny $\mathrm{P}^{4}$, Goncalvesova $\mathrm{E}^{4}$, Schonrath $\mathrm{F}^{5}$, Gummert $\mathrm{J}^{6}$ \\ Medical Faculty of the Comenius University, National Institute of Cardiovascular Diseases, \\ Department of Cardiac Surgery, Bratislava, Slovakia. panayiotisartemiou@yahoo.com
}

\begin{abstract}
OBJECTIVES: Mechanical circulatory support is an established therapy in end-stage heart failure. The EUROMACS registry was created to promote research in these patients. The aim of this report was to present our 12 year experience with the durable mechanical circulatory support devices and compare it with the EUROMACS registry.

METHODS: Data from the entire EUROMACS registry from January 2011 to April 2019 were included (4704 implantations in 4410 patients). During the 12 years of our experience, until April 2019,125 mechanical support devices were implanted, in 122 patients. We compare patients' characteristics, operative data and results with the EUROMACS registry and we report the major complications during the observational period. RESULTS: Primary end-point (death) occurred in 40 (32.8\%) patients in our cohort during the followup period, representing the survival rate $75 \%, 68 \%$, and $58 \%$ for $6,12,24$ months respectively, which compares favourably with the data, reported by the EUROMACS registry, the survival $66 \%$ and $53 \%$ after 1 and 2 years respectively. Cerebrovascular accident occurred in $7 \%$, a bleeding event in $32 \%$, significant infection (driveline) in $78 \%$ and a device malfunction in $13 \%$ of the patients. Forty- three patients underwent a heart transplant with hospital and long-term mortality of $11.6 \%$ and $14 \%$ respectively.

CONCLUSION: Mechanical circulatory support is a valuable therapeutic option with excellent survival rates, nevertheless it is associated with clinically significant complications rates.

The direct comparison between our cohort and the EUROMACS registry showed that early implantation strategy and mini invasive approach may improve survival rates and decrease postoperative complications (Tab. 3, Fig. 3, Ref. 16). Text in PDF www.elis.sk

KEY WORDS: durable mechanical circulatory support, EUROMACS registry.
\end{abstract}

\section{Introduction}

Long-term mechanical circulatory support (MCS) is an actively developing field in modern cardiac surgery and cardiology. International registries have been established to enhance scientific

'Medical Faculty of the Comenius University, National Institute of Car-
diovascular diseases, Department of Cardiac Surgery, Bratislava, Slova-
kia, ${ }^{2}$ EUROMACS, European Association for Cardio-Thoracic surgery,
Windsor, United Kingdom, ${ }^{3}$ Department of Cardiothoracic Surgery Eras-
mus University Medical Center, Rotterdam, the Netherlands, ${ }^{4}$ Medical
Faculty of the Comenius University, National Institute of Cardiovascu-
lar diseases, Department of Cardiac Surgery, Bratislava, Slovakia, ${ }^{5}$ De-
partment of Cardiothoracic and Vascular Surgery, German Heart Centre,
DZHK (German Centre for Cardiovascular Research), Partner Site Berlin,
Berlin, Germany, and ${ }^{6}$ Herz und Diabeteszentrum Nordrheim-Westfalen,
Bad Oeynhausen, Germany

Address for correspondence: P. Artemiou, MD, Medical Faculty of the Comenius University, National Institute of Cardiovascular Diseases, Department of Cardiac Surgery, Pod krasnou horkou 1, SK-831 01 Bratislava, Slovakia.

Phone: +421.917665774 , Fax: +421.259320287

Acknowledgements: The European Association for Cardio-Thoracic Surgery, EACTS, supported this work. insights, to address safety concerns and to implement the standard of care in the patients receiving MCS.

The Interagency Registry for Mechanically Assisted Circulatory Support (INTERMACS) was created for North America in 2005 by the Food and Drug Administration (FDA) and the National Heart, Lung and Blood Institute (NHLB), with mandatory participation of all the centres implanting durable MCS in USA. The 8th annual report published in 2017, summarized implantation experiences involving 22866 MCS devices including 373 total artificial hearts (TAHs) in adult patients between June 2006 and 31 December 2016, making it the largest reported cohort to date (1).

The European Registry for Mechanically Assisted Circulatory Support (EUROMACS) was established in 2009 and at the end of April 2019, EUROMACS comprised 52 centres from 18 countries. Its international internet platform (Dendrite Clinical Systems Ltd) allows data entry for adults and paediatric patients implanted with durable ventricular assist devices (VAD) or TAHs that have been designed for prolonged MCS of longer than 6 months. Up to now, 4400 adult patients are registered and 2 summarizing reports were published (2-5). EUROMACS is a part of the International register for Mechanical Assisted Circulatory Support (IMACS), which collects data globally (6). 
Tab. 1. Demographic data.

\begin{tabular}{|c|c|c|c|c|c|}
\hline \multirow{2}{*}{ Variable } & \multicolumn{2}{|c|}{ EUROMACS } & \multicolumn{3}{|c|}{ BRATISLAVA $n=122$} \\
\hline & Mean, $n, \%$ & $\mathrm{CI}$ & Mean, $n, \%$ & $\mathrm{CI}$ & $\mathrm{p}$ \\
\hline Age (years) & 53.4 & $18-86$ & 49 & $47-51$ & 0.9669 \\
\hline Female gender & $774(16.99 \%)$ & $18-83$ & $14(11 \%)$ & $6-16$ & 0,1081 \\
\hline Ethnic origin & $\mathrm{n}=4555$ & & & & \\
\hline African American or black & $18(0.4 \%)$ & $0.3-0.5$ & 0 & 0 & $>0.9999$ \\
\hline Asian & $276(6.1 \%)$ & $5.5-6.6$ & 0 & 0 & 0.0013 \\
\hline Caucasian & $33345(73.4 \%)$ & $71.2-74.6$ & $122(100 \%)$ & 0 & $<0.0001$ \\
\hline Hawaian or other pacific islander & $3(0.1 \%)$ & $0.03-0.17$ & 0 & 0 & $>0.9999$ \\
\hline Unknown & $913(20 \%)$ & $19-21$ & 0 & 0 & $<0.0001$ \\
\hline Weight (kg) & 81 & $35-190$ & 83 & $80-86$ & 0.9934 \\
\hline Body Surface Area $\left(\mathrm{m}^{2}\right)$ & 1.83 & $1.29-3.07$ & 2.06 & $2.01-2.11$ & 0.9339 \\
\hline Body Mass Index $\left(\mathrm{kg} / \mathrm{m}^{2}\right)$ & 25.5 & $12.9-62.8$ & 26.7 & $25.9-27.5$ & 0.9877 \\
\hline BNP preoperatively $(\mathrm{pg} / \mathrm{ml})$ & $\mathrm{n} / \mathrm{a}$ & $\mathrm{n} / \mathrm{a}$ & 11239 & $8384-14094$ & \\
\hline Primary diagnosis & $\mathrm{n}=4212$ & & & & \\
\hline Idiopathic dilated $\mathrm{CM}$ & $958(22.8 \%)$ & $21.6-24.2$ & $49(40.2 \%)$ & $31.6-48.8$ & $<0.0001$ \\
\hline Ischemic CM & $1358(32.2 \%)$ & $31.8-33.6$ & $44(36.1 \%)$ & $27.6-44.6$ & 0.3734 \\
\hline Myocarditis & $192(4.5 \%)$ & $3.9-5.1$ & $10(8.2 \%)$ & $3.4-13$ & 0.0602 \\
\hline Congenital heart disease & $42(1 \%)$ & $0.7-1.3$ & $1(0.8 \%)$ & $0-2.3$ & 0.8454 \\
\hline Dilated cardiomyopathy familiar & $94(2.2 \%)$ & $1.8-2.6$ & $5(4.1 \%)$ & $0.6-0.7$ & 0.1737 \\
\hline Coronary heart disease & $438(10.4 \%)$ & $9.5-12.3$ & $1(0.8 \%)$ & $0-2.3$ & 0.0005 \\
\hline Cancer & $9(0.2 \%)$ & $0.1-0.3$ & 0 & 0 & $>0.9999$ \\
\hline Dilated cardiomyopathy post partum & $20(0.5 \%)$ & $0.3-0.7$ & 0 & 0 & $>0.9999$ \\
\hline Dilated cardiomyopathy toxic & $76(1.8 \%)$ & $1.2-2.0$ & $2(1.6 \%)$ & $0-3.8$ & 0.8925 \\
\hline Dilated cardiomyopathy viral & $25(0.6 \%)$ & $0.4-0.8$ & $2(1.6 \%)$ & $0-3.8$ & 0.1478 \\
\hline Hypertrophic cardiomyopathy & $48(1.2 \%)$ & $0.9-1.5$ & $3(2.5 \%)$ & $0-5$ & 0.1828 \\
\hline Unknown & $848(20.1 \%)$ & $18.9-21.3$ & 0 & 0 & $<0.0001$ \\
\hline Other & $104(2.5 \%)$ & $2-3$ & $5(4.1 \%)$ & $0.6-7.6$ & 0.2572 \\
\hline \multicolumn{6}{|l|}{ Comorbidities } \\
\hline Frequent flyer profile & $\mathrm{n} / \mathrm{a}$ & $\mathrm{n} / \mathrm{a}$ & $13(10 \%)$ & $5-15$ & \\
\hline Temporary circulatory support & $477(11 \%)$ & $10.1-11.9$ & $16(13 \%)$ & $7-19$ & 0.5393 \\
\hline Haemodialysis & $130(0.3 \%)$ & $0.25-0.35$ & $2(1.6 \%)$ & $0-3.8$ & 0.3592 \\
\hline ICD device in place & $\mathrm{n} / \mathrm{a}$ & $\mathrm{n} / \mathrm{a}$ & $99(81 \%)$ & $74-88$ & \\
\hline Diabetes mellitus & $1056(25 \%)$ & $24-26$ & $23(18 \%)$ & $11-25$ & 0.1174 \\
\hline Insulin dependent & $286(7 \%)$ & $6.3-7.7$ & $11(47 \%)$ & $27-67$ & 0.3373 \\
\hline Cerebrovascular event & $\mathrm{n} / \mathrm{a}$ & $\mathrm{n} / \mathrm{a}$ & $18(14 \%)$ & $8-20$ & \\
\hline Symptomatic PAD & $\mathrm{n} / \mathrm{a}$ & $\mathrm{n} / \mathrm{a}$ & $1(0.8 \%)$ & $0-2.3$ & \\
\hline Carotid artery disease & $\mathrm{n} / \mathrm{a}$ & $\mathrm{n} / \mathrm{a}$ & $1(0.8 \%)$ & $0-2.3$ & \\
\hline \multicolumn{6}{|l|}{ Medical therapy prior to implant } \\
\hline Aspirin & $1849(44 \%)$ & $43-45$ & $34(28 \%)$ & $20-36$ & 0.0004 \\
\hline ACE inhibitor & $1573(37 \%)$ & $36-38$ & $47(39 \%)$ & $31-47$ & 0.7908 \\
\hline $\mathrm{ARB}$ & $568(13 \%)$ & $12-14$ & $14(11 \%)$ & $6-16$ & 0.521 \\
\hline b-blocker & $1768(42 \%)$ & $41-43$ & $81(68 \%)$ & $60-76$ & $<0.0001$ \\
\hline Aldosterone antagonist & $2190(52 \%)$ & $21-53$ & $100(84 \%)$ & $78-90$ & $<0.0001$ \\
\hline iv inotropes immediately prior to implant & $\mathrm{n} / \mathrm{a}$ & $\mathrm{n} / \mathrm{a}$ & $86(70 \%)$ & $78-94$ & \\
\hline Current device therapy & $\mathrm{n}=4655$ & & & & \\
\hline Bridge to recovery & $103(2 \%)$ & $1.6-2.4$ & 0 & 0 & 0.1147 \\
\hline Bridge to candidancy & $1502(33 \%)$ & $32-34$ & $38(31 \%)$ & $23-39$ & 0.7929 \\
\hline Bridge to transplant & $1647(36 \%)$ & $35-37$ & $70(57 \%)$ & $49-65$ & $<0.0001$ \\
\hline Destination therapy & $753(17 \%)$ & $16-18$ & $9(7 \%)$ & $3-11$ & 0.0088 \\
\hline Rescue therapy & $303(7 \%)$ & $6.3-7.7$ & $5(4 \%)$ & $0.5-7.5$ & 0.2845 \\
\hline Other and unknown & $247(5 \%)$ & $0.4-5.6$ & 0 & 0 & 0.0028 \\
\hline
\end{tabular}

$\mathrm{CM}=$ cardiomyopathy, $\mathrm{ICD}=$ implantable cardioverter defibrillator, $\mathrm{PAD}=$ peripheral artery disease, $\mathrm{ACE}=$ angiotensin converting enzyme, $\mathrm{ARB}=$ angiotensin receptor blocker, $\mathrm{n} / \mathrm{a}=$ non-available, data not sent by EUROMACS, $\mathrm{CI}=$ confidence interval

Our Institute participates in the EUROMACS registry, and the aim of this report was to present our 12 year experience with the durable mechanical circulatory support devices and compare it with the EUROMACS registry.

\section{Patients and methods}

\section{EUROMACS registry}

In EUROMACS, the anonymized patient baseline, follow-up and adverse events are transmitted from participating sites using 
a secure, web-based system. All centres agreed that their data be made available for scientific analyses.

\section{Study population}

Data from the entire EUROMACS registry with the implantation date from January 2011 to April 2019 were included in this study, forming a cohort of 4704 implantations in 4410 patients. During the 12 years of experience in our institute until April 2019,125 MCS, mainly VAD and TAHs were implanted, in 122 patients. Our institute is a member of the EUROMACS registry since the year 2019, and up to February 2019, data to the EUROMACS registry were submitted retrospectively. Data from our centre is not included in the overall EUROMACS data analysed, and these are two independent patient cohorts. The annual mean number of LVAD implantations was 11.5 (2-21), and in terms of case number our centre belongs to the group of intermediate size centres that contribute data to the EUROMACS registry.

We used patients' charts to collect demographic, operative and postoperative data. Death or serious adverse events (major infection, major bleeding, neurological complications-cerebrovascular accident and device malfunction) were all reported in the database. Also, minor incidents (cardiac arrhythmia, pericardial fluid collection, haemolysis, hepatic dysfunction, hypertension, psychiatric episode, renal dysfunction, respiratory failure, right heart failure, arterial non-CNS thromboembolism, wound dehiscence, venous thromboembolism and myocardial infarction), were also reported in the database.

In this report, we compare patients' characteristics, operative data and results of a single centre (National Institute of Cardiovascular Diseases, Bratislava, Slovakia) with the entire EUROMACS registry and we report the major complications during the observational period.

Both, the EUROMACS registry and our cohort are comparable in terms of age, gender, primary diagnosis, INTERMACS level and preoperative hemodynamic.

Written informed consent, and an approval of the ethics committee were obtained for the submission of clinical data to the EUROMACS registry from all our patients, as well as for the publication of this report.

\section{Primary and secondary end-points}

All-cause mortality was the primary end-point of this study. Secondary end-points were serious adverse events.

Major infection was defined as clinically relevant if antibiotic administration or surgical intervention was required.

Any bleeding into a critical organ (cerebral, pericardial), irrespective of its magnitude or of bleeding in any other location, that required transfusion of at least two units of packed red blood cells or other intervention was considered major.

Tab. 2. Imaging and haemodynamic data.

\begin{tabular}{|c|c|c|c|c|c|}
\hline \multirow{2}{*}{ Variable } & \multicolumn{2}{|l|}{ EUROMACS } & \multicolumn{3}{|c|}{ BRATISLAVA $\mathrm{n}=122$} \\
\hline & Mean, n, \% & $\mathrm{CI}$ & Mean, n, \% & $\mathrm{CI}$ & $\mathrm{p}$ \\
\hline Left ventricular systolic ejection fraction & $\mathrm{n}=3166$ & & & & \\
\hline Very severely reduced (LVEF $\leq 19 \%)$ & $1514(48 \%)$ & $47-49$ & $100(81 \%)$ & $75-87$ & $<0.0001$ \\
\hline Severely reduced $(\mathrm{LVEF}=20-29 \%)$ & $1396(44 \%)$ & $43-45$ & $21(17 \%)$ & $11-23$ & $<0.0001$ \\
\hline Moderately reduced $(\mathrm{LVEF}=30-39 \%)$ & $194(6 \%)$ & $5.2-6.8$ & $1(0.8 \%)$ & $0-2.3$ & 0.0149 \\
\hline Mildly reduced $(\mathrm{LVEF}=40-50 \%)$ & $35(1.2 \%)$ & $0.9-1.5$ & 0 & 0 & 0.6396 \\
\hline \multicolumn{6}{|l|}{ New York Functional Class } \\
\hline Class I & $\mathrm{n} / \mathrm{a}$ & $\mathrm{n} / \mathrm{a}$ & 0 & 0 & \\
\hline Class II & $\mathrm{n} / \mathrm{a}$ & $\mathrm{n} / \mathrm{a}$ & 0 & 0 & \\
\hline Class III & $\mathrm{n} / \mathrm{a}$ & $\mathrm{n} / \mathrm{a}$ & $9(7 \%)$ & $3-11$ & \\
\hline Class IV & $\mathrm{n} / \mathrm{a}$ & $\mathrm{n} / \mathrm{a}$ & $113(93 \%)$ & $88-98$ & \\
\hline INTERMACS level & $\mathrm{n}=4181$ & & & & \\
\hline 1 (cardiogenic shock) & $668(16 \%)$ & $15-17$ & $11(9 \%)$ & $4-14$ & 0.0376 \\
\hline 3 (Inotrope-dependent) & $1081(26 \%)$ & $25-27$ & $36(39 \%)$ & $21-37$ & 0.3643 \\
\hline 4 (resting symptoms) & $721(17 \%)$ & $16-18$ & $22(18 \%)$ & $12-14$ & 0.8204 \\
\hline 5 (exertion intolerant) & $159(3.8 \%)$ & $3.3-4.3$ & $5(4 \%)$ & 1.7 & 0.8666 \\
\hline 6 (exertion limited) & $57(1.4 \%)$ & $1-1.6$ & $3(2.5 \%)$ & $0-5$ & 0.309 \\
\hline 7 (advanced NYHA Class III) & $135(3.6 \%)$ & $3.1-4.1$ & $6(5 \%)$ & $2-8$ & 0.3016 \\
\hline \multicolumn{6}{|l|}{ Haemodynamics } \\
\hline Heart rate & 86 & $40-218$ & 84 & $82-86$ & 0.994 \\
\hline Systolic blood pressure (mmHg) & 100 & $33-192$ & 100 & $98-102$ & $>0.9999$ \\
\hline PA systolic blood pressure (mmHg) & 65 & $10-139$ & 64 & $60-68$ & 0.9959 \\
\hline PCWP $(\mathrm{mmHg})$ & $\mathrm{n} / \mathrm{a}$ & $\mathrm{n} / \mathrm{a}$ & 30 & $29-31$ & \\
\hline Pulmonary vascular resistance (dyn.s/ $\left.\mathrm{cm}^{5}\right)$ & $\mathrm{n} / \mathrm{a}$ & $\mathrm{n} / \mathrm{a}$ & 436 & $354-518$ & \\
\hline Cardiac index $\left(1 / \mathrm{min} / \mathrm{m}^{2}\right)$ & 2.04 & $0.52-3.82$ & 1.7 & $1.55-1.85$ & 0.8871 \\
\hline TAPSE $(\mathrm{mm})$ & 15 & $1-197$ & 13 & $12-14$ & 0.9945 \\
\hline
\end{tabular}

$\overline{\mathrm{LVEF}}=$ left ventricular ejection fraction, $\mathrm{NYHA}=$ New York Heart Association, $\mathrm{PA}=$ pulmonary artery, $\mathrm{PCWP}=$ pulmonary capillary wedge pressure, TAPSE $=$ tricuspid annular plane systolic excursion, $\mathrm{n} / \mathrm{a}=$ non-available, data not sent by EUROMACS, CI $=$ confidence interval 
Tab. 3. Operative and postoperative data.

\begin{tabular}{|c|c|c|c|c|c|}
\hline \multirow{2}{*}{ Variable } & \multicolumn{2}{|c|}{ EUROMACS n=4824 } & \multicolumn{3}{|c|}{ BRATISLAVA n=122 } \\
\hline & Mean, n, \% & $\mathrm{CI}$ & Mean, $n, \%$ & $\mathrm{CI}$ & $\mathrm{p}$ \\
\hline CPB time (min) & 114 & $6-612$ & 91 & $85-97$ & 0.9811 \\
\hline Off-pump & $370(9.6 \%)$ & $\mathrm{n} / \mathrm{a}$ & 0 & 0 & \\
\hline \multicolumn{6}{|l|}{ Device type } \\
\hline LVAD & $3111(64 \%)$ & $63-65$ & $112(91 \%)$ & $87-95$ & $<0.0001$ \\
\hline LVAD off-pump & $781(16 \%)$ & $15-17$ & 0 & 0 & $<0.0001$ \\
\hline BiVAD & $162(3 \%)$ & $2.5-3.5$ & 0 & 0 & 0.0343 \\
\hline BiVAD off-pump & $4(0.08 \%)$ & $0.07-0.09$ & 0 & 0 & $>0.9999$ \\
\hline LVAD/RVAD & $144(2.9 \%)$ & $2.5-3.3$ & $6(5 \%)$ & $2-8$ & 0.2189 \\
\hline LVAD/RVAD off-pump & $21(0.4 \%)$ & $0.3-0.5$ & 0 & 0 & $>0.9999$ \\
\hline RVAD & $322(6 \%)$ & $5.3-6.7$ & $3(2.5 \%)$ & $0-5$ & 0.0634 \\
\hline RVAD off-pump & $95(1.9 \%)$ & $1.6-2.2$ & 0 & 0 & 0.1755 \\
\hline TAH & $184(3.8 \%)$ & $3.3-4.3$ & $4(3 \%)$ & 0.6 & 0.76 \\
\hline ICU/CCU stay & 22 & $0-830$ & 19 & $15-23$ & 0.9982 \\
\hline Step-down care stay & $\mathrm{n} / \mathrm{a}$ & $\mathrm{n} / \mathrm{a}$ & 15 & $14-16$ & \\
\hline
\end{tabular}

$\mathrm{CPB}=$ cardiopulmonary bypass, $\mathrm{LVAD}=$ left ventricular assist device, $\mathrm{BiVAD}=$ biventricular assist device, $\mathrm{RVAD}=$ right ventricular assist device, $\mathrm{TAH}=$ total artificial heart, ICU = intensive care unit, $\mathrm{CCU}=$ cardiac care unit

Loss of function of any vital part of the implanted devices mechanical system (pump, controller, cable) posing a threat to the patients' health or life, requiring change in management or exchange was interpreted as device malfunction.

Cerebrovascular accident was defined as any transient or permanent neurological deficit in clinical or imaging studies believed to be caused by a central nervous abnormality (haemorrhagic or ischemic stroke, transient ischemic accident, epileptic event).

\section{Statistical analysis}

All variables were expressed as median, $95 \%$ confidence intervals, and qualitative variables as numbers and percentages. Kaplan-Meier estimates of cumulative probabilities were calculated for the primary (death) and secondary end-points using the entire EUROMACS registry and the Bratislava cohort. The Kaplan-Meier curves include $95 \%$ confidence intervals. Some EUROMACS data are missing from the tables because it was not possible to retrieve them from the database. Numeric variables were analysed with Student's t-test. Chi square test or Fisher's exact test were performed for categorical variables. Statistical significance was considered for $\mathrm{p}<0.05$.

\section{Results}

\section{Baseline characteristics}

The baseline characteristics of the patients in both groups are presented in Tables 1 and 2. At the time of analysis, the EUROMACS registry included 4655 implantations, of which 125 patients were recruited from Bratislava. The mean age in our cohort was 49 years, while in the EUROMACS registry it was 53 years Fewer female patients and patients with larger body surface area were observed in our cohort, $11 \%$ vs $17 \%$ and $2.06 \mathrm{~m}^{2}$ vs 1.82 $\mathrm{m}^{2}$ respectively. Body mass index of the patients in both cohorts was almost similar.

In our cohort, idiopathic dilated cardiomyopathy, followed by ischemic cardiomyopathy were the leading causes for heart fail- ure, $(40.2 \%$ vs $22.8 \%, \mathrm{p}<0.0001)$, while in the EUROMACS registry the leading cause for heart failure was ischemic cardiomyopathy followed by idiopathic dilated cardiomyopathy (32.2\% vs $36.1 \%, p=0.3734)$.

New York Heart Association class (NYHA) and INTERMACS profile were the parameters used to assess the optimal timing for implantation. In our cohort, $93 \%$ of the patients were in NYHA class IV. Concerning the INTERMACS profile of the patients, data were comparable. In ours and EUROMACS cohort, most patients were considered as level 2 (progressive decline) (32\% vs $33 \%$, $\mathrm{p}=0.8527$ ) or level 3 (stable, but inotropic dependent) (39\% vs $26 \%, p=0.3643)$. Finally, in the EUROMACS registry, more patients were in INTERMACS level $1(16 \%$ vs $9 \%$, p = 0.0376).

Implantation strategies appear to differ between the cohorts. VADs were used as bridge-to-transplant (36\%), bridge-to-candidacy (33\%), bridge to recovery (2\%), destination therapy (17\%),

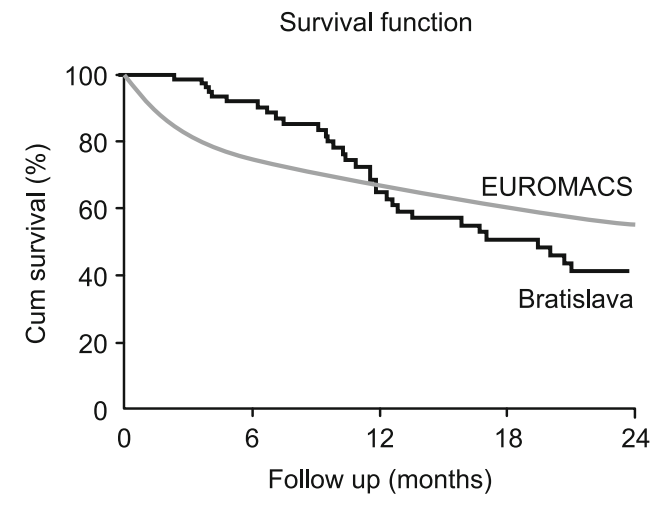

\begin{tabular}{|l|c|c|c|c|c|}
\hline \multicolumn{7}{|c|}{ Number at risk } \\
\hline Months & 0 & 6 & 12 & 18 & 24 \\
\hline Bratislava & 110 & 56 & 36 & 23 & 16 \\
\hline EUROMACS & 2321 & 1384 & 1016 & 721 & 498 \\
\hline
\end{tabular}

Fig. 1. Kaplan-Meier survival analysis of the Bratislava cohort compared with the entire EUROMACS cohort. 


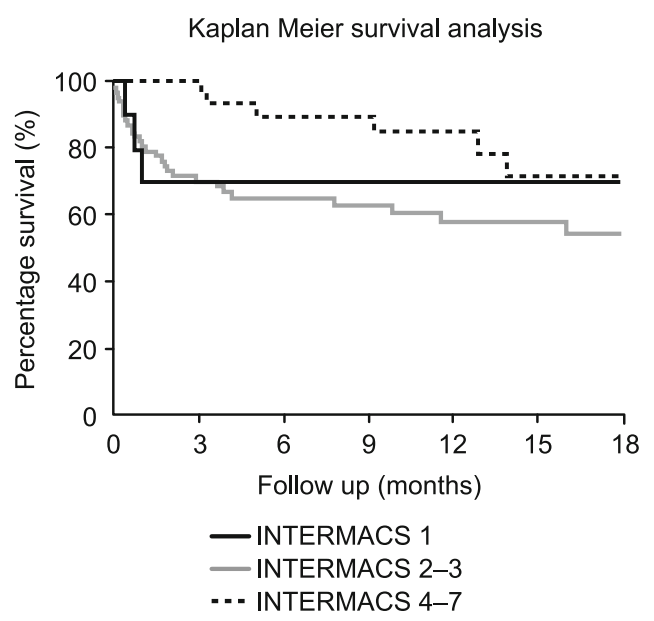

Fig. 2. Kaplan-Meier survival analysis of the Bratislava cohort dependent on the INTERMACS status at the time of implantation.

rescue therapy ( $7 \%$ ) and other causes (5\%) in the EUROMACS cohort, while in our cohort, they were used as bridge-to-transplant $(57 \%, \mathrm{p}<0.0001)$, bridge-to-candidacy $(31 \% \mathrm{p}=0.7929)$, destination or rescue therapy in $7 \%(p=0.0088)$ and $4 \%(p=$ 0.2845 ) respectively.

Significant differences in medical therapy prior to VAD implantation were observed. Beta-blockade and aldosterone antagonist therapy was less used in the EUROMACS cohort (42\% vs $68 \%, \mathrm{p}<0.0001$ and $52 \%$ vs $84 \%, \mathrm{p}<0.0001$ respectively), while aspirin use was more in the EUROMACS cohort ( $44 \%$ vs $28 \%, p=0.0004$ ) and corresponded with the higher number of patients with ischemic cardiomyopathy.

\section{Operative and postoperative data}

In our cohort, cardiopulmonary bypass was used for the VAD implantation, and the mean time was 23 minutes less compared to the EUROMACS cohort. No difference in the use of left ventricular assist devices and TAHs between the EUROMACS and our cohort was observed ( $80 \%$ vs $91 \%, 3.8 \%$ vs $3 \%$ ) respectively. The stay in the intensive care unit was similar in both cohorts (Tab. 3).

\section{Survival analysis}

Kaplan-Meier estimates the survival in our cohort at 6 months $75 \%$ (CI 65.7-82.4), at 12 months $68 \%$ (CI 57.8-77.3), at 18 months $61 \%$ (CI 49.2-72.1), at 2 years $58 \%$ (CI 44.9-69.8) and at 3 years $43 \%$ (CI 18-67.8) (Fig. 1). During the follow-up period, 43 patients underwent heart transplant with a hospital and long-term mortality (April 2019) of $11.6 \%$ (5 patients) and $14 \%$ (6 patients) respectively. The median waiting period time was 470.8 (53-1352) days.

Kaplan-Meier analysis of our cohort, grouped by INTERMACS level prior to implantation, demonstrated a correlation between INTERMACS level and both early ( $<30$ days) and longterm survival. INTERMACS level 1 and 2 exhibited the high early mortality, and might stabilization afterwards, but the number of the patients at risk is small. INTERMACS levels 4-7 have better early and long-term survival (Fig. 2).

\section{Follow-up}

The median follow-up and MCS support time was 14 (2-66) months.

\section{Complications in the Bratislava cohort}

Freedom from device malfunction at 2 years was $96 \%$ (CI 87.9-98.7), and after the second year fell to $88 \%$ (CI 62.6-96.9), resulting in 0.11 events per patient year. The most frequent device malfunction was device thrombosis that was treated mainly conservatively, followed by controller, which was replaced. One patient had pump exchange because he denied anticoagulation treatment.

Most infections occurred during the early period. The most frequent infection was, driveline infection ( $78 \%$ of the patients), followed by bronchopneumonia. Two patients had positive blood cultures, most probably due to device-related infection, and both patients were treated successfully conservatively. Freedom from infection at first, second and third year was $94 \%$ (CI 83.8-98.2), $77 \%$ (CI 56.7-89.5) and $54 \%$ (CI 26.6-75.3) respectively, resulting in 0.22 events per patient year. No patient underwent the pump exchange due to infection.

Freedom from neurologic complications during follow-up was $97.5 \%$ (CI 91.6-99.5), resulting in 0.06 events per patient year. Intracranial bleeding was observed in 5 patients, and stroke occurred in 4 patients. Two patients died as the result of the intracranial bleeding.

In our cohort, the incidence of late bleeding complications during the follow-up period was low and was observed in 4 patients, resulting in 0.02 events per patient year. Gastrointestinal bleeding occurred in 3 patients. Bleeding events of the central nervous system are discussed separately. On the other hand, $32 \%$ of the patients had re-exploration due to postoperative bleeding, representing 10.9 events per 100 patients-month during the first three months after a device implantation. In our cohort, we observed a significant decline in the post-opertative bleeding complications after the year 2014. At that that time, we introduced the mini invasive approach through upper mini-sternotomy and left minithoracotomy. There was a decline from $48.9 \%$ (22 patients from 45 ) to $18.9 \%$ (17 patients from 97).

The corresponding Kaplan-Meier estimates are depicted in Figure 3.

\section{Discussion}

Survival

Primary end-point (death) occurred in 40 (32.8\%) of the patients in our cohort over the entire period of follow-up, representing the survival rate of $75 \%, 68 \%$, and $58 \%$ for $6,12,24$ months respectively. This compares favourably with the data of the 2 nd EUROMACS annual report, which reported the survival of $66 \%$ after 1 year and $53 \%$ after 2 years. On contrary, data from the 8 th INTERMACS annual report (1) and the 2nd IMACS annual report (6) showed 1 -and 2-year survival of $81 \%, 70 \%$ and $80 \%$ and $70 \%$ 


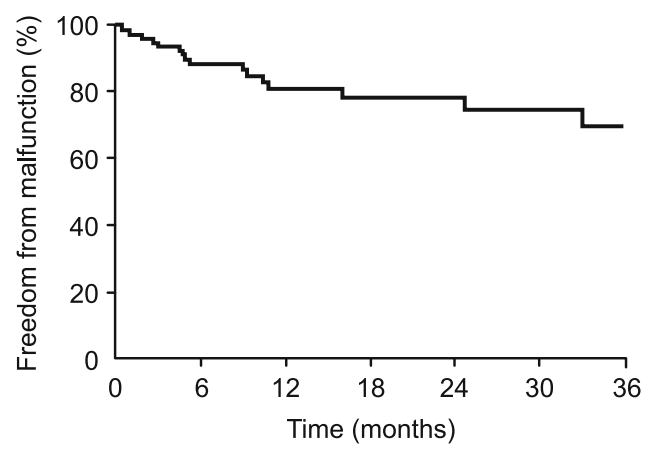

\begin{tabular}{|l|c|c|c|c|c|c|}
\hline \multicolumn{7}{|c|}{ Number at risk } \\
\hline All patients & 60 & 38 & 27 & 22 & 16 & 14 \\
\hline Time (months) & 6 & 12 & 18 & 24 & 30 & 36 \\
\hline
\end{tabular}

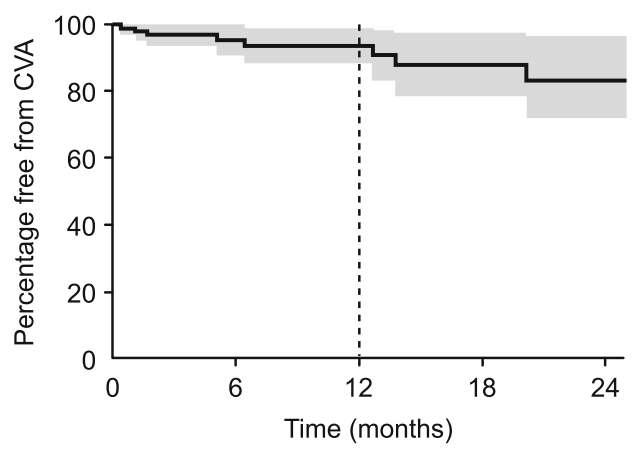

\begin{tabular}{|l|c|c|c|c|c|}
\hline \multicolumn{5}{|c|}{ Number at risk } \\
\hline All patients & 110 & 56 & 34 & 23 & 16 \\
\hline Time (months) & 0 & 6 & 12 & 18 & 24 \\
\hline
\end{tabular}

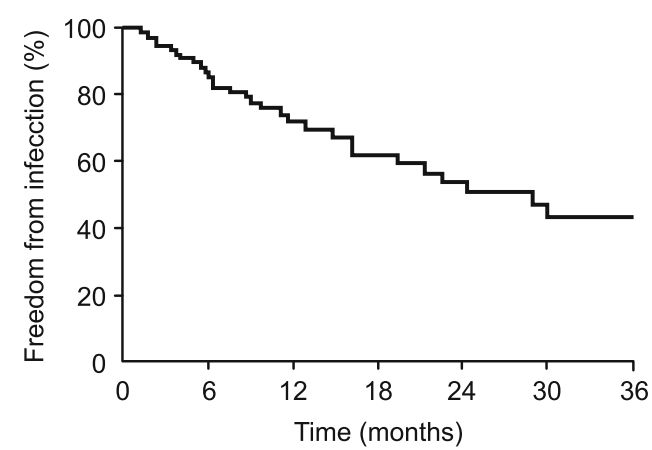

\begin{tabular}{|l|c|c|c|c|c|c|}
\hline \multicolumn{7}{|c|}{ Number at risk } \\
\hline All patients & 63 & 37 & 27 & 22 & 16 & 14 \\
\hline Time (months) & 6 & 12 & 18 & 24 & 30 & 36 \\
\hline
\end{tabular}

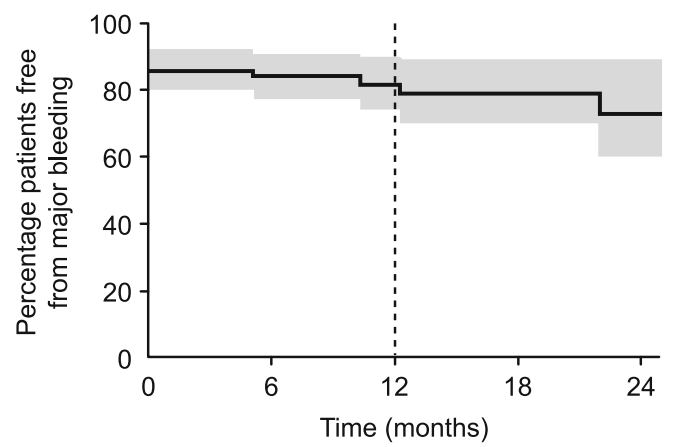

\begin{tabular}{|l|c|c|c|c|c|}
\hline \multicolumn{5}{|c|}{ Number at risk } \\
\hline All patients & 117 & 51 & 26 & 18 & 12 \\
\hline Time (months) & 0 & 6 & 12 & 18 & 24 \\
\hline
\end{tabular}

Fig. 3. Time of first event analysis of (A) device malfunction, (B) clinically significant infections, (C) cerebrovascular accident (CVA), and (D) major bleeding of the Bratislava cohort.

respectively. In our cohort, an improvement was observed also in the postoperative results in terms ICU stay after the introduction of the minimal invasive approach in 2014. Since then, 42 patients had a VAD implantation with this procedure. Favourable results with the use of the minimal invasive approach in VAD implantation are also reported by other authors $(7,8)$.

Moreover, although, in our institute we are in favour of the early implantation strategy in relatively stable patients, which may improve survival rates, our data tend to indicate that our patients were generally of more advanced INTERMACS stage, with severely reduced LVEF ( $81 \%$ patients with LVEF less than $20 \%$ ). This is related to disease pathology and status of the patients at initial presentation.

\section{Complications}

During the follow-up period in our cohort, the most prevalent complication was infection, and according to the most recent INTERMACS report (1), it was still the fourth most common cause of death within 1 year after implant. In our cohort, $78 \%$ of the patients had driveline infections. Driveline infections increased the mortality (9), but with proper measures like earlier detection, frequent dressing changes, local antiseptics, prolonged or lifelong antibiotics, surgical revision and emergency transplantation, the patients might have a favourable outcome (10). Finally, no patient underwent the pump exchange due to infection. Data from the 2nd EUROMACS annual report (3) showed that the incidence of infections at 3 months was 5.49 events per 100 patients per year. On contrary, data from the IMACS registry showed that the incidence of infections at 3 months was 3.51 events per 100 patients per year (6).

The recently reported incidence of cerebrovascular accident after VAD implantation in the IMACS registry was $19 \%$. In the recently published comparison of neurological outcomes between the recipients of HeartWare (Medtronic) and HeartMate II (Abbott), complications were reported in $19 \%$ for 0.44 median years of follow-up and $16 \%$ for 0.95 median years of followup. Advancing age was found to be a risk factor for any adverse neurologic outcome (12). Other risk factors for cerebrovascular accident included international normalized ratio (INR) level, aortic cross clamping, stroke in the past and postoperative infection 
(13). In the 2nd EUROMACS annual report (3), the incidence of the neurologic complications at 3 months was reported to be 1.87 events per 100 patients per month. In our cohort, the incidence of neurologic complications was relatively low, $7 \%$ of the patients, resulting in 0.06 events per patient year.

The incidence of pump malfunction in our cohort was $13 \%$, resulting in 0.11 events per patient year, and the main reason was pump thrombosis. During the whole follow-up period, only one patient needed the pump exchange due to thrombosis and the rest of the patients were successfully treated conservatively. Moreover, two patients had the control unit replacement due to malfunction, and in one patient with TAH, a mechanical malfunction of the Freedom driver system controller due to mechanical damage appeared, which was managed successfully by the controller change. Data from the 2nd EUROMACS annual report (3) showed that the incidence of pump malfunction at 3 months was $14.7 \%$, resulting in 2.88 events per 100 patient months. According to the MOMENTUM 3 trial (14), the incidence of pump thrombosis was lower in HeartMate 3 (Abbott) compared to the HeartMate II (Abbott). In our cohort, during the follow-up in patients with HeartMate 3 (Abbott) VAD we did not observe any pump thrombosis.

Data from our cohort showed that $32 \%$ of the patients, resulting in 10.9 events per 100 patients-months during the first 3 months, had re-exploration due to severe postoperative bleeding. There was a significant decline in postoperative bleeding complications after the year 2014 and the introduction of the mini invasive approach through upper mini-sternotomy and left mini-thoracotomy, from $48.9 \%$ to $18.9 \%$ ( 0.03 events per 100 patients-months Vs 0.013 events per 100 patients-months). The incidence of postoperative bleeding in the EUROMACS, INTERMACS and IMACS registries at 3 months were, 6.45 events per 100 patients-month, 16.24 events per 100 patients-months, and $35 \%$ of the patients, resulting in 13.78 events per 100 patients-months respectively (1, $3,6)$. On contrary, in our cohort, we observed a lower incidence of post-operative bleeding complications compared to the INTERMACS, IMACS and EUROMACS registries. The introduction of the mini invasive approach through the upper mini-sternotomy and left mini-thoracotomy, had a significant role in this incidence decline of the postoperative bleeding complication. Similar results with the use of the minimal invasive approach in VAD implantation are also reported by other authors $(7,8)$.

The standard medical therapy consists of acetylsalicylic acid $100 \mathrm{mg}$ daily and warfarin with target INR 2-3.5. The patients were all well educated about warfarin treatment, they had frequent INR controls and the dose was adjusted appropriately, they all used CoaguCheckXS (Roche Diagnostics, GmbH), and the INR level was kept on the lower therapeutic level. All these factors might contribute to the low incidence of bleeding complications in our cohort. Moreover, gastrointestinal bleeding was the most prevalent bleeding complication after continuous flow VAD implantation as the result of the creation of arteriovenous malformations and acquired von Willebrand factor deficiency, and published data presented up to $26.6 \%$ of the patients had this complication $(15,16)$. In the EUROMACS registry (3), the incidence of gastrointestinal bleeding events after 3 months from implantation was 1.86 events per 100 patients-month. In the eighth annual INTERMACS report (1), the incidence of gastrointestinal bleeding reported was 7.09 events per 100 patients-month axial flow pumps and 5.26 per 100 patients -months for centrifugal flow pumps. The longer the mechanical circulatory support, the more frequent were the bleeding complications. In our cohort, the mean circulatory support time was 426 days and 3 patients had a gastrointestinal bleeding, with an incidence of 0.02 events per patient per year.

\section{Conclusion}

We conclude that international registries provide valuable data, which may in turn, lead to benchmarking new insights, approaches and discussions. Consequently, this will lead to quality improvement. Comparing local with international data is clearly feasible and comparison of the single centre experience with the EUROMACS database generates interesting observations and shows differences in the approaches and outcomes of the MCS therapy. In our institute, we are in favour of the early implantation strategy in relatively stable patients, which may improve the survival rates. However, the rate of complications after MCS implantation remains considerable. The take home message from this direct comparison between our cohort and the EUROMACS registry is that early implantation strategy and mini invasive approach may improve the survival rates and decrease postoperative complications such as postoperative bleeding.

\section{Limitations}

This is mainly a retrospective report and some data may not be complete. Also, some data could not be provided by the EUROMACS registry. The primary diagnosis in $20 \%$ of the patients, as well as the NYHA clinical status, PCWP and pulmonary vascular resistance for all EUROMACS patients is not known. This would seem to demonstrate some of the limitation of registry data. On the other hand, the missing details do not significantly reduce the importance of being able to analyse a large number of cases, especially for hard outcomes like mortality. Moreover, in our centre, we do not perform off-pump assist device implantation, so we do not have any data to compare with the EUROMACS registry. Finally, in our cohort, only 4 patients received a TAH, 6 a BiVAD and 3 an RVAD, and in this way bias may be caused by the larger number of BiVAD, RVAD and TAH patients in the EUROMACS registry.

The EUROMACS registry continues recruiting to increase the numbers of contributing centres, the goal being to include as many European centres as possible. In contrast to the situation in the USA, participation in EUROMACS is not mandatory in EUROPE. Therefore, surveillance and improvement of data quality are ongoing efforts.

\section{References}

1. Kirklin JK, Pagani FD, Kormos RL et al. Eighth annual INTERMACS report: Special focus on framing the impact of adverse events. J Heart Lung Transplant 2017; 36 (10): 1080-1086. 


\section{1-378}

2. de By TMMH, Mohacsi P, Gahl B et al. The European Registry for patients with Mechanical Circulatory Support (EUROMACS): first annual report. Eur J Cardiothorac Surg 2015; 47: 770-777.

3. de By TMMH, Mohacsi P, Gahl B et al. The European Registry for patients with Mechanical Circulatory Support (EUROMACS) of the European Association for Cardio-Thoracic Surgery (EACTS): second report. Eur J Cardiothorac Surg 2018; 53: 309-316.

4. Zuk k, Gahl B, Susak M et al. Mid-term mechanical circulatory support: comparison of single-center data with the EUROMACS registry. Eur J Cardiothorac Surg 2017; 51: 127-135.

5. de By TMMH, Waheed H, Berger F et al. The European Registry for patients with Mechanical Circulatory Support (EUROMACS): first EUROMACS Paediatric (Paedi-EUROMACS) report. Eur J Cardiothorac Surg 2018; 54: 800-808.

6. Kirklin JK, Xie R, Cowger $\mathbf{J}$ et al. Second annual report from the ISHLT Mechanically Assisted Circulatory Support (IMACS) registry. J Heart Lung Transplant 2018; 37: 685-691.

7. Wachter K, Franke UFW, Rustenbach CJ, Baumbach H. Minimally invasive versus conventional LVAD-implantation-An analysis of the literature. Thorac Cardiovasc Surg 2019; 67 (3): 156-163.

8. Mohite PN, Sabashnikov A, Raj B et al. Minimally invasive left ventricular assist device implantation: a comparative study. Artif Organs 2018; 42 (12): 1125-1131.

9. Koval CE, Thuita L, Moazami N, Blackstone E. Evolution and impact of drive-line infection in a large cohort of continuous-flow ventricular assist device recipients. J Heart Lung Transplant 2014; 11: 1164-1172.
10. Potapov EV, Antonides C, Crespo-Leiro MG et al. 2019 EACTS expert consensus on long-term mechanical circulatory support. . Eur J Cardiothorac Surg 2019; 56: 230-270.

11. Givertz MM, DeFilippis EM, Colvin M et al. HFSA/SAEM/ISHLT clinical expert consensus document on the emergency management of patients with ventricular assist devices. J Heart Lung Transplant 2019; 38: 677-698.

12. Coffin ST, Haglund NA, Davis XE et al. Adverse neurologic events in patients bridged with long-term mechanical circulatory support: A device-specific comparative analysis. J Heart Lung Transplant 2015; 34 (12): 1578-1585.

13. Tsiouris A, Heliopoulos I, Mikroulis D, Mitsias PD. Stroke after implantation of continuous flow left ventricular assist devices. J Car Surg 2019; 34: 541-548.

14. Mehra MR, Uriel N, Naka $Y$ et al. A fully magnetically levitated left ventricular assist device-Final report. N Engl J Med 2019; 380 (17): 1618-1627.

15. Kawabori M, Kurihara C, Critsinelis AC et al. Gastrointestinal bleeding after HeartMate IIor HVAD implantation: Incidence, location etiology and effect on survival. ASAJO J 2019 Apr 4 doi: 10.1097/ MAT.0000000000000998.

16. Kataria R, Jorde UP. Gastrointestinal bleeding during continuousflow left ventricular assist device support: State of the field Cardiol Rev 2019; 27 (1): 8-13. DOI: 10.1097//CRD.000000000000212.

Received November 10, 2020. Accepted November 25, 2020. 